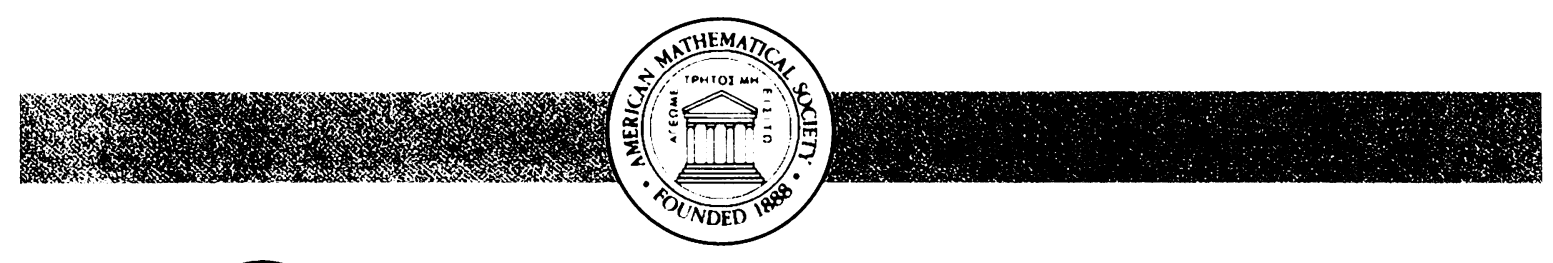

\title{
CONTEMPORARY MATHEMATICS
}

\section{6}

\section{Logic and \\ Computation}

Proceedings of a Workshop held at

Carnegie Mellon University, June 30-July 2, 1987

\section{Wilfried Sieg, Editor}




\title{
EDITORIAL BOARD
}

\author{
Daniel M. Burns, Jr., managing editor \\ Richard W. Beals \\ Gerald J. Janusz \\ Sylvain E. Cappell \\ Jan Mycielski \\ David Eisenbud \\ Michael E. Taylor \\ Jonathan Goodman
}

The Workshop on Logic and Computation was held at Carnegie Mellon University on June 30-July 2, 1987.

1980 Mathematics Subject Classification (1985 Revision). Primary 03, 68.

\section{Library of Congress Cataloging-in-Publication Data}

Workshop on Logic and Computation (1987: Carnegie Mellon University)

Proceedings of the Workshop on Logic and Computation: proceedings of a conference held June 30-July 2, 1987, at Carnegie Mellon University/Wilfried Sieg, editor.

p. cm.-(Contemporary mathematics, ISSN 0271-4132; v. 106)

ISBN 0-8218-5110-1 (alk. paper)

1. Computable functions-Data processing-Congresses. I. Sieg, Wilfried, 1945-. II. Title. III. Series: Contemporary mathematics (American Mathematical Society); v. 106.

QA9.59.W67 1987

$511.3-\mathrm{dc} 20$

Copying and reprinting. Individual readers of this publication, and nonprofit libraries acting for them, are permitted to make fair use of the material, such as to copy an article for use in teaching or research. Permission is granted to quote brief passages from this publication in reviews, provided the customary acknowledgment of the source is given.

Republication, systematic copying, or multiple reproduction of any material in this publication (including abstracts) is permitted only under license from the American Mathematical Society. Requests for such permission should be addressed to the Executive Director, American Mathematical Society, P.O. Box 6248, Providence, Rhode Island 02940-6248.

The appearance of the code on the first page of an article in this book indicates the copyright owner's consent for copying beyond that permitted by Sections 107 or 108 of the U.S. Copyright Law, provided that the fee of $\$ 1.00$ plus $\$ .25$ per page for each copy be paid directly to the Copyright Clearance Center, Inc., 27 Congress Street, Salem, Massachusetts 01970. This consent does not extend to other kinds of copying, such as copying for general distribution, for advertising or promotional purposes, for creating new collective works, or for resale.

Copyright (C) 1990 by the American Mathematical Society. All rights reserved.

The American Mathematical Society retains all rights except those granted to the United States Government.

Printed in the United States of America.

This volume was printed directly from author-prepared copy.

The paper used in this book is acid-free and falls within the guidelines established to ensure permanence and durability. @

Portions of this publication were typeset using AMS -TEX, the American Mathematical Society's TEX macro system.

10987654321959493929190 


\section{Contents}

Preface

WILFRIED SIEG

Some theories conservative over intuitionistic arithmetic

MICHAEL BEESON

Ramsey interpreted: A parametric version of Ramsey's theorem GIANLUIGI BELLIN

Notions of closed subsets of a complete separable metric space in weak subsystems of second order arithmetic

DOUGLAS K. BROWN

A note on polynomial time computable arithmetic

WILFRIED BUCHHOLZ and WILFRIED SIEG

Axiomatizations and conservation results for fragments of bounded arithmetic

SAMUEL R. BUSS

A smash-based hierarchy between PTIME and PSPACE

PETER G. CLOTE

Polymorphic typed lambda-calculi in a type-free axiomatic framework SOLOMON FEFERMAN

Polynomial time computable arithmetic

FERNANDO FERREIRA

Metaprogramming in SIL

CHRIS GOAD

$W K L_{0}$ and orderings of countable abelian groups

KOSTAS HATZIKIRIAKOU and STEPHEN G. SIMPSON

Marriage theorems and reverse mathematics

JEFFRY L. HIRST 
Computationally based set existence principles

DANIEL LEIVANT

Hierarchy results for mixed-time

KEN MCALOON

Polynomial time equivalence types

A. NERODE and J. B. REMMEL

Program development through proof transformation

FRANK PFENNING

Some models of Scott's theory $L C F$ based on a notion of rate of convergence

RICK STATMAN

Sharply bounded arithmetic and the function $a-1$

GAISI TAKEUTI

Radon-nikodym theorem is equivalent to arithmetical comprehension XIAOKANG YU 


\title{
A NOTE ON POLYNOMIAL TIME COMPUTABLE ARITHMETIC
}

\author{
Wilfried Buchholz and Wilfried Sieg
}

\begin{abstract}
In Ferreira's contribution to these Proceedings the class $\mathscr{P}$ of polynomial time computable functions is characterized as the class of provably recursive functions of some weak formal theories. The first such characterization of $\mathscr{P}$ was given, of course, by Buss. A form of Herbrand's theorem for partially normalized derivations is used in this note to obtain Ferreira's results. Such "Herbrand-analyses" have been applied in a variety of contexts (see [F/S],[L], [S1]): they are most appropriate if one wants to extract computational information from derivations; they are conceptually clear and technically strong.
\end{abstract}

A. INTRODUCTION. The class $\mathscr{P}$ of polynomial time computable functions is characterized in [F] as the class of provably recursive functions of three restricted theories for binary trees or 0 -1-words. The basic theory, PTCA, allows induction for polynomial time decidable predicates; $\mathrm{PTCA}^{+}$is obtained from it by expanding the induction schema to NP-predicates. The third theory, $\left(\Sigma_{1}^{\mathrm{b}}-\mathrm{PIND}\right)$, is like $\mathrm{PTCA}^{+}$, but its language contains only symbols for some basic functions, not for all elements of $\mathscr{P}$. That the latter theory has exactly the elements of $\mathscr{P}$ as its provably recursive functions is the analogue of the main theorem in [B] for $n=1$. Ferreira obtains this result by a mixture of model- and proof- theoretic techniques. We give a canonical, purely proof-theoretic Herbrand-analysis that yields Ferreira's result for $\mathrm{PTCA}^{+}$and brings nut most sharply the central problem; namely, the analysis of weak induction schemata by recursive functions of low complexity.

The main ideas for this paper emerged in the summer of 1988, when we gave a joint seminar at the Ludwigs-Maximilians-Universität in Muinchen. Buchholz presented [F] in the seminar; Sieg was working on his [S2] in which Herbrand-analyses for systems of (bounded) arithmetic are given. So it was natural to explore whether they can be given for Ferreira's theories of binary trees. Our note is thus complementing [F].

B. BOUNDED LOGICAL COMPLEXITY. We use the same formal framework as $[\mathrm{F}]$; in particular, $L$ is the first order language with constant symbols $\varnothing, 0,1$, function symbols $\wedge$ and $x$, and two binary relation symbols $\subseteq$ and $=$. The language $L(\mathscr{P})$, i.e. $L_{p}$ in $[F]$, is obtained from $L$ by adding function symbols for each element of $\mathscr{P}$. The

\section{Mathematics Subject Classification (1985 Revision). O3F30}


latter class can be defined inductively as the smallest class $X$ of functions, such that $X$ contains certain initial functions $\left(Z ; P_{i}^{n}, 1 \leq i \leq n, n \in \mathbb{N} ; C_{0}, C_{1}\right.$, and $\left.Q\right)$, and is closed under composition and bounded iteration. Let $s t$ abbreviate $s^{\wedge} t$ for L-terms $s$ and $t$ : $\left.x\right|_{z}=y$ stands for $(1 \times z \subseteq 1 \times x \& y \subseteq x \& 1 \times z=1 \times y) \vee(1 \times x \subseteq 1 \times z \& y=x)$. Using these abbreviations we formulate the schema of bounded iteration :

$f$ is defined by iteration from $g, h_{0}, h_{1}$ with bound $t[x, y]$ if

$$
\begin{aligned}
& f(x, \varnothing)=g(x) \\
& f(x, y i)=\left.h_{i}(x, y, f(x, y))\right|_{t[x, y]} \quad(i=0,1)
\end{aligned}
$$

where $t$ is an L-term and $\mathbf{X}$ indicates a possibly empty sequence of variables. As we are going to work in a Tait-style sequent calculus, it is convenient to build up formulas from literals (atomic or negations of atomic formulas) by using $\&, \vee, \forall, \exists$. Negations of complex formulas, conditionals and biconditionals are defined as usual.

\section{DEFINITIONS}

1.1. $Q F(\mathscr{P})$ denotes the set of quantifier-free formulas of $L(\mathscr{P})$.

1.2. $(\forall x \subseteq y) \varphi[(\exists x \subseteq y) \varphi]$ abbreviates $(\forall x)(x \subseteq y \rightarrow \varphi)[(\exists x)(x \subseteq y \& \varphi)$, resp.].

1.3. $(\exists x \leq t) \varphi$ abbreviates $(\exists x)(x \leq t \& \varphi)$, where $s \leq t$ is $1 \times s \subseteq 1 \times t$.

1.4. A formula $\varphi$ is in $\Delta_{0}^{\mathrm{b}}(\mathscr{P})\left[\Sigma_{1}^{\mathrm{b}}(\mathscr{P})\right.$, resp.] if it has been obtained from literals in $\mathrm{L}(\mathscr{P})$ by $\&, \vee, \forall \subseteq, \exists \subseteq,[$ and $\exists s$, resp.].

1.5. An $L(\mathscr{P})$-formula $\varphi$ is in $s-\Sigma_{1}^{b}(\mathscr{P})$ if it is of the form $(\exists y s t) \psi$ with $\psi \in Q F(\mathscr{P})$.

The formulas in $\Delta_{0}^{\mathrm{b}}(\mathscr{P})$ are exactly the polynomial time computable matrices of [F]. The theories for binary trees to be investigated contain the basic axioms for the non-logical symbols of $L$ (see $[F]$ ), the defining equations for the elements of $\mathscr{P}$ in case the theory is formulated in $L(\mathscr{P})$, and the induction principle on notations for classes of formulas $\mathscr{F}: \varphi \emptyset \&(\forall x)(\varphi x \rightarrow \varphi \times 0 \& \varphi \times 1) \rightarrow(\forall x) \varphi x(\varphi \in \mathscr{F})$. The latter schema is denoted by $\mathscr{F}-\mathrm{NIA}$; the resulting theory - always with classical logic - is called $(F-N I A)$.

We formulate a few properties of $\mathscr{P}$ that are provable in $(\mathrm{QF}(\mathscr{P})-\mathrm{NIA})$.

\section{LEMMA.}

(i) For every term $s$ of $L(\mathscr{P})$ there is a term $t$ of $L$, such that $(Q F(\mathscr{P})-N I A)$ proves $s \leq t$.

(ii) For any $\varphi_{1}(\mathbf{x}), \ldots, \varphi_{n}(\mathbf{x}) \in Q F(\mathscr{P})$ and $f_{1}, \ldots, f_{n+1} \in \mathscr{P}$ there is an $f \in \mathscr{P}$ such that $(\mathrm{QF}(\mathscr{P})-\mathrm{NIA})$ proves

$$
\begin{aligned}
\left(\varphi_{1}(\mathbf{x}) \& f(\mathbf{x})=f_{1}(\mathbf{x})\right) & \vee\left(\neg \varphi_{1}(\mathbf{x}) \& \varphi_{2}(\mathbf{x}) \& f(\mathbf{x})=f_{2}(\mathbf{x})\right) \\
& \vee\left(\neg \varphi_{1}(\mathbf{x}) \& \neg \varphi_{2}(\mathbf{x}) \& \varphi_{3}(\mathbf{x}) \& f(\mathbf{x})=f_{3}(\mathbf{x})\right) \\
& \vdots \\
& \vee\left(\neg \varphi_{1}(\mathbf{x}) \& \ldots \& \neg \varphi_{n}(\mathbf{x}) \& f(\mathbf{x})=f_{n+1}(\mathbf{x})\right) .
\end{aligned}
$$

(iii) For any $\varphi(x, y) \in Q F(\mathscr{P})$ there is an $h \in \mathscr{P}$, such that $(Q F(\mathscr{P})-$ NIA) proves

$$
\cdot((\exists y \subseteq \mathrm{x}) \varphi(\mathbf{x}, \mathrm{y}) \leftrightarrow \varphi(\mathbf{x}, \mathrm{h}(\mathbf{x}, \mathrm{x}))) .
$$

The last part of the lemma allows us to prove that in $\left(Q F(\mathscr{P})\right.$-NIA) every $\Delta_{0}^{b}(\mathscr{P})$-formula is equivalent to a quantifier-free formula; proposition 6 of [F] establishes in turn that in $\left(s-\Sigma_{1}^{b}(\mathscr{P})-N I A\right)$ every $\Sigma_{1}^{b}(\mathscr{P})$-formula is equivalent to one in $s-\Sigma_{1}^{b}(\mathscr{P})$. 
Thus we have:

3. PROPOSITION.

(i) $\left(\Delta_{0}^{\mathrm{b}}(\mathscr{P})-\mathrm{NIA}\right)$ is equivalent to $(\mathrm{QF}(\mathscr{P})-\mathrm{NIA})$.

(ii) $\left(s-\Sigma_{1}^{b}(\mathscr{P})-N I A\right)$ is equivalent to $\left(\Sigma_{1}^{b}(\mathscr{P})-N I A\right)$.

Notice that $\left(\Delta_{0}^{b}(\mathscr{P})-\mathrm{NIA}\right)$ is Ferreira's PTCA, and $\left(s-\sum_{1}^{b}(\mathscr{P})-\mathrm{NIA}\right)$ is his PTCA ${ }^{+}$. Now we turn our attention to bounding the complexity of formulas in derivations. The latter are now presented in a Tait-style calculus as in [Sch]; the induction principle is given equivalently by a rule $F-\mathrm{NIR}^{*}$ of the form

$$
\frac{\Delta, \neg \varphi \times, \varphi \times 0 \quad \Delta, \neg \varphi \times, \varphi \times 1}{\Delta, \neg \varphi \emptyset, \varphi s} \quad(\varphi \in \mathscr{F})
$$

where $s$ is a term, and $x$ must not occur in the lower sequent. This new formulation of $(\mathscr{F}$-NIA) has two virtues - it is equivalent to the earlier one and allows us to prove partial normalization theorems.

\section{DEFINITION .}

A derivation in $(F-N I A)$ is called I-normal if and only if all its cuts are either I-cuts or have atomic cut-formulas; where a cut with cut-formula $\varphi$ is called an I-cut if one of its premises is the conclusion of the induction rule with principal formula $\varphi$ or $\neg \varphi$.

The standard proof of the normalization theorem for predicate logic can readily be adapted to show that any derivation in $(\mathscr{F}-\mathrm{NIA})$ can be I-normalized.

6. THEOREM. (I-normalization) If $D$ is a derivation of $\Gamma$ in $(F-N I A)$. then there is an $I$-normal derivation $D^{\circ}$ of the same endsequent in $(F-N I A)$.

The length $\left|D^{\circ}\right|$ of $D^{\circ}$ can be bounded by $2 m, m=\rho(D)-1$; the cut-rank function $\rho$ takes into account only the complexity of cuts that are not I-cuts. I-normal derivations do not have the subformula property, but the complexity of formulas occurring in them can nevertheless be bounded significantly.

7. COROLLARY. Let $\mathscr{F}$ and $\mathscr{G}$ be classes of formulas that are closed under substitution. If $D$ is an I-normal derivation of $\Gamma$ in $(F-N I A)$ with $\Gamma \subseteq \mathscr{G}$, then any formula in $D$ is either atomic or a subformula of an element of $\mathscr{F} \cup\{\neg \varphi: \varphi \in \mathscr{F}\} \cup \mathscr{G}$.

\section{EXTRACTING TERMS.}

The I-normalization theorem will be used to establish a (generalized) Herbrand-theorem.

8. THEOREM. ( $\exists$-inversion) Let $\Gamma$ contain only existential formulas, and let $\psi$ be quantifier-free; if $\Gamma,(\exists y) \psi y$ is provable in $\left(Q F(\mathscr{P})\right.$-NIA) then there is a term $t^{*}$ such that $\Gamma, \psi \mathrm{t}^{*}$ is also provable in $(\mathrm{QF}(\mathscr{P})-\mathrm{NIA})$. ( $\psi$ may contain additional variables.)

PROOF. The proof proceeds by induction on I-normal (QF( $\mathscr{P})$-NIA)-derivations $D$. We focus on the central step, when $N^{*}{ }^{*}$ is the last rule applied in $D$. (The other non-trivial cases, e.g. $\exists$-introduction, require definition by cases.) 
Then $D$ is of the form

$$
\mathrm{D}\left\{\mathrm{D}_{0}\{\underbrace{}_{=\Gamma} \frac{\mid \underbrace{}_{\Delta, \neg \varphi \mathrm{x}, \varphi \mathrm{x} 0,(\exists \mathrm{y}) \psi \mathrm{y} \quad \Delta, \neg \varphi \mathrm{x}, \varphi \mathrm{x} 1,(\exists \mathrm{y}) \psi \mathrm{y}},(\exists \mathrm{y}) \psi \mathrm{y}}{\Delta_{1}, \neg \varnothing, \varphi s} \mathrm{D}_{1}\right.
$$

The induction hypothesis applied to the $D_{i}$ yields terms $t_{i}[x]$ and derivations $D_{i}^{*}$ of

$$
\Delta, \neg \varphi \times, \varphi \times \mathrm{i}, \psi \mathrm{t}_{\mathrm{i}}[\mathrm{x}] \quad(\mathrm{i}=0,1) .
$$

Obviously ( $Q F(\mathscr{P})-\mathrm{NIA})$ proves

$$
\neg \varphi \emptyset, \varphi s, \exists \mathrm{x} \subseteq s[\varphi \times \& \neg(\varphi \times 0 \& \varphi \times 1)] .
$$

By Lemma 2 (iii) there is an $h \in \mathscr{P}$, such that $(\mathrm{QF}(\mathscr{P})$-NIA) proves

(3) $\neg \exists \mathrm{x} \subseteq \mathrm{s}[\varphi \times \& \neg(\varphi \times 0 \& \varphi \times 1)], \varphi \mathrm{h}(\mathrm{s}) \& \neg(\varphi \mathrm{h}(\mathrm{s}) 0 \& \varphi \mathrm{h}(\mathrm{s}) 1)$.

From (2) and (3) we obtain:

(4) $\neg \varphi \emptyset, \varphi s, \varphi \mathrm{h}(\mathrm{s})$,

$$
\neg \varphi \emptyset, \varphi s, \neg \varphi \mathrm{h}(s) 0, \neg \varphi \mathrm{h}(\mathrm{s}) 1 \text {. }
$$

From (1) (with $h(s)$ substituted for $x$ ) and (5) we obtain $\neg \varphi \emptyset, \varphi s, \Delta, \neg \varphi \mathrm{h}(\mathrm{s}), \psi \mathrm{t}_{0}[\mathrm{~h}(\mathrm{~s})], \psi \mathrm{t}_{1}[\mathrm{~h}(\mathrm{~s})]$ and then (by (4)) $\Delta, \neg \varphi \emptyset, \varphi s, \psi t_{0}[h(s)], \psi t_{1}[h(s)]$.

This together with Lemma $2($ ii) gives us an $f \in \mathscr{P}$ such that $(Q F(\mathscr{P})$-NIA) proves $\Delta, \neg \varphi \emptyset, \varphi s, \psi \mathrm{f}(\mathrm{s})$. Q.E.D.

The 3 -inversion is crucial for establishing the main conservation result.

9. THEOREM. $\left(s-\Sigma_{1}^{b}(\mathscr{P})-\mathrm{NIA}\right)$ is conservative over $(Q F(\mathscr{P})-\mathrm{NIA})$ with respect to $\Pi_{2}^{0}$-sentences $\varphi$ of the form $(\forall x)(\exists y) \varphi^{*}(x, y)$ with $\varphi^{*} \in Q F(\mathscr{P})$.

PROOF. As $(\mathrm{QF}(\mathscr{P})-\mathrm{NIA})$ is contained in $\left(s-\sum_{1}^{b}(\mathscr{P})-\mathrm{NIA}\right)$ we have to show only that every $\Pi_{2}^{0}$-sentence provable in the latter theory is provable in the former. This is achieved by transforming any I-normal derivation $D$ in $\left(s-\Sigma_{1}^{b}(\mathscr{P})-N I A\right)$ of a sequent $\Delta$. where $\Delta$ contains only existential formulas, into a derivation $D^{\prime}$ of $\Delta$ in $(Q F(\mathscr{P})-N I A)$. We proceed by induction on the number $*$ of applications of $\operatorname{NIR}^{*}$ in $D$, not counting for sure $N I R^{*}$-instances with formulas in $Q F(\mathscr{P})$. - The case $\sharp=0$ is trivial. So let be $m+1$ and consider an uppermost instance of $N I R^{*}$ with $\psi$ of the form $(\exists y)(y \leq t[x]$ $\left.\& \psi^{*} \mathrm{yx}\right)$, where $\psi^{*}$ is in $\mathrm{QF}(\mathscr{P})$; both $\psi^{*}$ and $\mathrm{t}$ may contain additional variables. The subderivation $E$ of $D$ determined by that inference is of the form

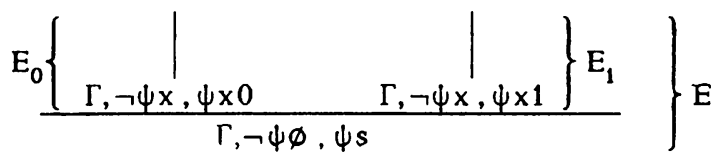

Taking into account the form of $\psi$ and the fact that $D$ is an I-normal derivation in $\left(s-\Sigma_{1}^{b}(\mathscr{P})-\mathrm{NIA}\right)$, we can obtain (recalling corollary 7 ) by repeated $\forall$-inversion from the $E_{i}$ derivations of $\Gamma^{*}, \neg\left(y \leq t[x] \& \psi^{*} y x\right), \psi x i$, where $\Gamma^{*}$ contains only existential formulas. $]$-inversion yields terms $t_{i}[y, x]$ and derivations in $(Q F(\mathscr{P})$-NIA) of

$\left(\square_{i}\right) \quad \Gamma^{*}, \neg\left(y \leq t[x] \& \psi^{*} y x\right), t_{i}[y, x] \leq t[x i] \& \psi^{*} t_{i}[y, x] x i$ 
Now we define a function $f$ by iteration with bound $t\left[x_{0}\right] t\left[x_{1}\right]$ (using Lemma $2(i)$ and (ii) to bring the definition into the required form): $f(y, \varnothing)=y, f(y, x i)=t_{i}[f(y, x), x]$. From the derivations leading to the $\square_{i}$ and this definition we get derivations of

$$
\Gamma^{*}, \neg\left(f(y, x) \leq t[x] \& \psi^{*} f(y, x) x\right), f(y, x i) \leq t[x i] \& \psi^{*} f(y, x i) x i
$$

and by $Q F(\mathscr{P})-\mathrm{NIR}^{*}$ of

$$
\Gamma^{*}, \neg\left(\mathrm{y} s \mathrm{t}[\varnothing] \& \psi^{*} \mathrm{y} \varnothing\right), \mathrm{f}(\mathrm{y}, \mathrm{s}) \leq \mathrm{t}[\mathrm{s}] \& \psi^{*} \mathrm{f}(\mathrm{y}, \mathrm{s}) \mathrm{s} \text {. }
$$

With a little bit of logic we finally obtain a derivation $E^{\prime}$ in $(Q F(\mathscr{P})-N I A)$ of $\Gamma, \neg \psi \emptyset, \psi s$. Replace $E$ in $D$ by $E^{\prime}$. The resulting derivation has only $m$ applications of $s-\Sigma_{1}^{b}(\mathscr{P})-N I R^{*}$ and the induction hypothesis yields the above claim. Q.E.D.

\section{CHARACTERIZING $\mathscr{P}$.}

Since for every $L(\mathscr{P})$-term $t[x]$ the function $\lambda x \cdot t[x]$ is in $\mathscr{P}$, theorem 8 implies that the provably recursive functions of $(\mathrm{QF}(\mathscr{P})-\mathrm{NIA})$ are exactly the polynomial time computable ones. Using also theorem 9 and proposition 4 we obtain the sought after characterization result.

10. THEOREM.

$\mathscr{P}$ is exactly the class of provably recursive functions of $\left(\Sigma_{1}^{b}(\mathscr{P})-\mathrm{NIA}\right)$.

\section{REMARKS.}

(i) [F] establishes that $\left(\Sigma_{1}^{b}(\mathscr{P})-\mathrm{NIA}\right)$ is a conservative extension of $\left(\Sigma_{1}^{b}-\mathrm{NIA}\right)$; thus the theorem holds also for the latter theory.

(ii) The Herbrand-analysis given in $\mathrm{C}$ is insensitive to extensions of the various theories by $\Pi_{1}^{0}$-sentences. Thus, the main results hold also for $\Pi_{1}^{0}$-extensions of the theories involved.

\section{REFERENCES}

[B] Samuel Buss, Bounded Arithmetic, Bibliopolis, Napoli, 1986.

[F/S] Solomon Feferman and Wilfried Sleg, "Proof theoretic equivalences between classical and constructive theories for analysis", Springer Lecture Notes in Mathematics, vol. 897, (1981), pp. 78-142.

[F] Fernando Ferretra, "Polynomial time computable arithmetic", this volume.

[L] Horst Luckhardt, "Herbrand-Analysen zweier Beweise des Satzes von Roth: polynomiale Anzahlschranken", to appear in the Journal of Symbolic Logic.

[Sch] Helmut Schwichtenberg, "Proof theory: some applications of cut-elimination", Handbook of Math.Logic, J. Barwise (ed.), NHPC, Amsterdam 1977, pp. 867-895.

[S1] Wilfried Sieg, "Fragments of arithmetic", Annals of Pure and Applied Logic, 28 (1985), pp. 33-71.

[S2] Wilfried Sieg, "Derivations as computations", manuscript.

Wilfried Buchholz

Mathematisches Institut

Ludwig-Maximilians-Universität

8000 MÜNCHEN 2, West-Germany
Wilfried Sieg

Department of Philosophy

Carnegie Mellon University

PITTSBURGH, PA 15213, USA 